\title{
Optimal conceptual design and vision-based control of a fruit harvesting robot
}

\author{
K. Saran Kumar*, \\ P.S. Shivarama Shankaran and \\ S. Rizwan Asif
}

\author{
Department of Mechanical Engineering, \\ B.S. Abdur Rahman University, \\ Vandalur, Chennai - 600 048, Tamil Nadu, India \\ Email: sarankumar@bsauniv.ac.in \\ Email: shivramaps@gmail.com \\ Email: rizasif96@gmail.com \\ *Corresponding author
}

\section{S. Karthick, I.A. Palani, Bhupesh Kumar Lad, Abhijeet Patil, Pratik Patil and Harsh Sharma}

\author{
Mechatronics and Instrumentation Laboratory, \\ Discipline of Mechanical Engineering, \\ Indian Institute of Technology, Indore, \\ Simrol, Indore - 453 552, \\ Madhya Pradesh, India \\ Email: karthicks.iiti@gmail.com \\ Email:palaniia@iiti.ac.in \\ Email: bklad@iiti.ac.in \\ Email: abhijeetpatil45@gmail.com \\ Email: prtptl.smilingcorpse@gmail.com \\ Email: sharmaharsh2308@gmail.com
}

\begin{abstract}
The main contribution of this paper is to develop a vision-based control of a robotic arm for the harvesting fruits. The camera fixed in the gripper pad enables to precisely locate the fruit and pluck it from the branch. Rigorous stability analysis is done to ensure the guaranteed performance of the closed loop system. The camera feedback locates the exact position of the fruit; this enables the controller to track a suitable and optimal path to reach the target by performing desirable transformations. The manipulator with five-DOF (RRPRR) is designed and optimised for the formulating simple control strategies. The finger-like built gripper is electrically actuated to provide the necessary force required in harvesting the fruit. Also an additional bellow kind of structure is specially designed and located below the gripper which helps to roll down the harvested fruit on to the storage container without damaging it. Numerical simulation analysis was carried out along with the design realisation to justify the context. The advancement in the field of agrionics has also been a source of inspiration in designing agricultural robots.
\end{abstract}


Keywords: robot design; vision-based control; harvesting robot; dynamics; trajectory tracking; agrionics; farmbot; intelligent machine; fruit harvester.

Reference to this paper should be made as follows: Saran Kumar, K., Shivarama Shankaran, P.S., Asif, S.R., Karthick, S., Palani, I.A., Lad, B.K., Patil, A., Patil, P. and Sharma, H. (2018) 'Optimal conceptual design and vision-based control of a fruit harvesting robot', Int. J. Intelligent Machines and Robotics, Vol. 1, No. 1, pp.45-59.

Biographical notes: K. Saran Kumar completed his Master's in Computer Integrated Manufacturing Engineering at College of Engineering Guindy from Anna University Chennai. Previously, he was working as Teaching Faculty in the Department of Mechanical Engineering at NIT Tiruchirappalli. Currently, he is working as an Assistant Professor in the Department of Mechanical Engineering at B.S. Adbur Rahman Crescent University. His areas of interest are mechatronics system design, sensors and actuators, modelling and analysis, process automation and intelligent control, soft robotics and biomechatronics system design.

P.S. Shivarama Shankaran is pursuing his fourth year in Mechanical Engineering in B.S. Abdur Rahman Crescent University. His areas of interest include robotics, mechatronics, design and analysis and photography.

S. Rizwan Asif is doing his BTech in Mechanical Engineering from B.S. Abdur Rahman Crescent University. He is currently in the final year of his engineering studies. He is the Head of Design and Analysis of SAE BAJA Team Crescar and currently working on chassis design and analysis of the vehicle. His areas of interest include product design and analysis, control system, embedded system and robotics.

S. Karthick completed his Master's in Control and Instrumentation Engineering at College of Engineering Guindy from Anna University Chennai. Previously, he was working as Teaching Faculty in the Department of Instrumentation and Control Engineering at NIT Tiruchirappalli and as an Assistant Professor in Electrical Engineering at National Institute of Technology Kurukshetra. Currently, he is working as a Research Fellow in Mechatronics and Instrumentation Laboratory at Indian Institute of Technology Indore. His areas of interest are instrument design, sensors and actuators, modelling and analysis, process automation, soft computing, smart systems and intelligent control, soft robotics and biomechatronics, IIoT and CPS.

I.A. Palani is working as an Associate Professor in the Discipline of Mechanical Engineering, IIT Indore and also the Head of the Discipline of Metallurgy Engineering and Material Science. He did his post-doctoral fellowship in Graduate School of Information Science and Electrical Engineering, Kyushu University, Japan. He completed his $\mathrm{PhD}$ from Department of Mechanical Engineering from IIT Madras, India. He has authored book chapters on $\mathrm{ZnO}$ nanocrystals and allied materials. His areas of interest include instrumentation and mechatronics system design, laser assisted micro processing, surface engineering, optomechatronics, smart materials and nano structures for functional device.

Bhupesh Kumar Lad received his $\mathrm{PhD}$ degree in the area of Reliability Engineering from the Department of Mechanical Engineering, Indian Institute of Technology Delhi, New Delhi, India, in 2010. He worked with GE Global Research Centre, Bangalore, India, as a Research Engineer from 2010 to 2011. $\mathrm{He}$ is currently an Associate Professor in the Discipline of Mechanical 


\begin{abstract}
Engineering at the Indian Institute of Technology Indore, India. $\mathrm{He}$ is a Regional Editor (South Asia) of the International Journal of Performability Engineering, USA. He is the author of the book Machine Tool Reliability (Hoboken, NJ, USA and Salem, MA, USA: Wiley and Scrivener, 2016). He is an investigator of various research projects funded by national and international funding agencies. His major research interests include smart manufacturing, reliability engineering, and prognostics. He received his Hamied-Cambridge Visiting Lecture Fellowship of the University of Cambridge, UK in 2016.
\end{abstract}

Abhijeet Patil graduated from IIT Indore in Mechanical Engineering. He is currently working in Bharat Petroleum Corporation Limited (BPCL). His areas of interest include control system, mechatronics and process control.

Pratik Patil graduated from IIT Indore in Mechanical Engineering. He headed the team of IIT Indore for 2017 RoboCon Event. His research interests are robotics, modelling and analysis, control design, soft computing, and system identification.

Harsh Sharma graduated from IIT Indore in Mechanical Engineering. He is currently a Quantitative Analyst at Quantile Analytics. His areas of interest include robotics, control theory, mechatronics and machine learning.

\title{
1 Introduction
}

The main objective of this robot is to pluck fruits, and to increase the productivity of the agricultural industry. An algorithm was developed to determine the near minimum time path of a robot manipulator, with known kinematics and dynamics parameters. The method was based on calculating the distance of a near minimum time path and then defining the sequence of the robot. It is also applied to the specific problem of defining the motions for a fruit-picking robot. This algorithm enables us to determine the near-optimal sequence of task points through which the arm should pass and to find the minimum-time path between two points. Owing to nonlinearity and closely coupled nature of the manipulators equations of motions, it is rather complicated problem to solve (Edan et al., 1991). Multiple robots can be used to reduce the human labour and increase production. Since each robot has to be custom-designed and adapted to the specific crop, the design methodology presented here is applied to any moderate weight fruit from trees. Factors of fruits such as size, shape, firmness are considered. The locations of the fruits are also determined using vision. The harvesting robot autonomously performed its harvesting task by executing the predefined module to detect and localise the fruit in the trees, and control system regulated the manipulator and the end-effectors to approach and pick the fruits (De-An et al., 2011). Considering the combination of the distance estimation for the gripper, the fruit estimation precision is more critical as the gripper of the manipulator moves closer to the canopy (Li et al., 2011a). Considering optimal path as the performance index, robotic path planning simulated by robotic toolbox proved that the performance index was achieved using the genetic algorithm optimisation. This method also produced efficient results for shorter paths than any other traditional algorithm ( $\mathrm{Li}$ and Shi, 2010). The decision theoretic approach using the trichromatic coefficient of red and green was effective in segmenting the fruit from the background. 
The methodology presented can be applied to determine performance for proposed applications and thus substantially reduce the research and development costs associated with new agricultural robots (Edan and Miles, 1994). The applicability of the developed systems engineering methods was demonstrated. Performance of a robotic melon harvester was simulated to quantify effects of type of robot, number of arms, multiple configuration, and actuator speeds. An algorithm developed for comparing different robots indicated that the Cartesian robot performed faster and was capable of reaching all fruit (Edan and Miles, 1994). Advances in technology systems encourage the business stakeholders to apply more automation into the process. Modern process automations have been impacted by the advent of new technology systems and the agriculture and food sector is an exception (Suprem et al., 2013). At each position arm, the system was calibrated to a zero level, then the unit measured the movement of the arm around the target (dates fruit in bunches). The system shows promising efficiency in such cases (Aljanobi et al., 2010). Manual harvesting offers the benefits of maximum product quality, but has the disadvantages of uncertain labour availability and a relatively low picking rate (Sanders, 2005). The vision system used in the automatic harvester aims to detect the fruits and provides the information of the location and distance from the fruits to the robotic controller. In vision recognition system, vision cameras are mainly the solution to communicate with environment. The major achievements of vision systems have increased the role of vision-based control in many autonomous systems (Li et al., 2011b). Sensing and control systems currently used in rapid characterisation of machine state and soil variables are reviewed, with primary emphasis on the soil state and secondary interest on machine state (Hamrita et al., 2000). The fruit sorting technologies are so much improved that image processing and near infrared analysis have been used for automation and precise fruit inspection (Kondo, 2003).

Figure 1 (a) Conceptual drafted model of the robot - front view (b) Conceptual drafted model of the robot - side view (see online version for colours)

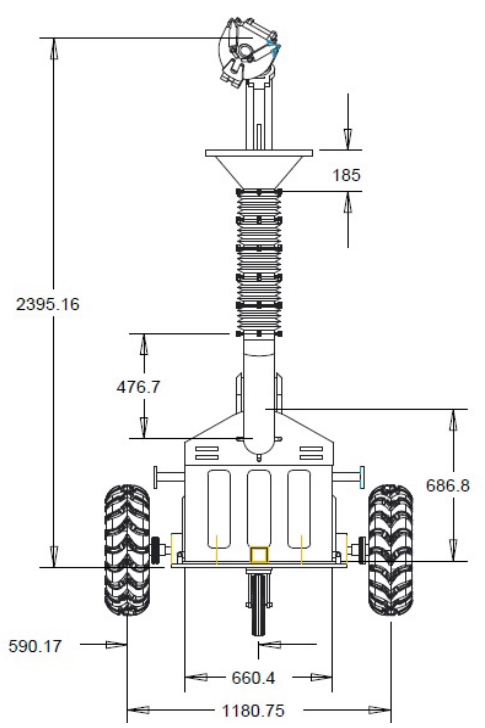

(a)

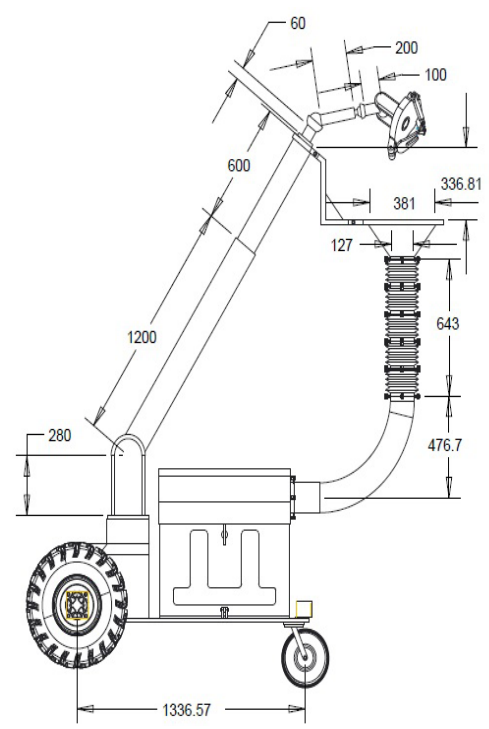

(b) 


\section{Design consideration and modelling}

A conceptual model of fruit harvesting robot was designed for both efficiency and low cost. The robotic harvester has five-DOF (RRPRR), it consists of a moving platform on which all the components are mounted. They include the manipulator, end-effectors attached with vision system (camera), actuators (drive unit), sensors and a bellows arrangement connecting the storage unit. The conceptual model of the system is shown in front and side view respectively in Figures 1(a) and (b).

\subsection{Mobile platform}

The moving platform is compactly designed, where the robot has two wheels at the rear end and a centre wheel at the front end. The rear wheels are powered by high torque motors and the front wheel has a freedom to rotate $360^{\circ}$ so it can adjust itself according to movement of the robot. The base of the robot is simple and lightweight making it more efficient to move around the agricultural field. There is a camera located at the front end of the manipulator which yaws about $180^{\circ}$ in order to identify the path or to avoid any abnormal disturbance in the path.

Figure 2 Manipulator design (see online version for colours)

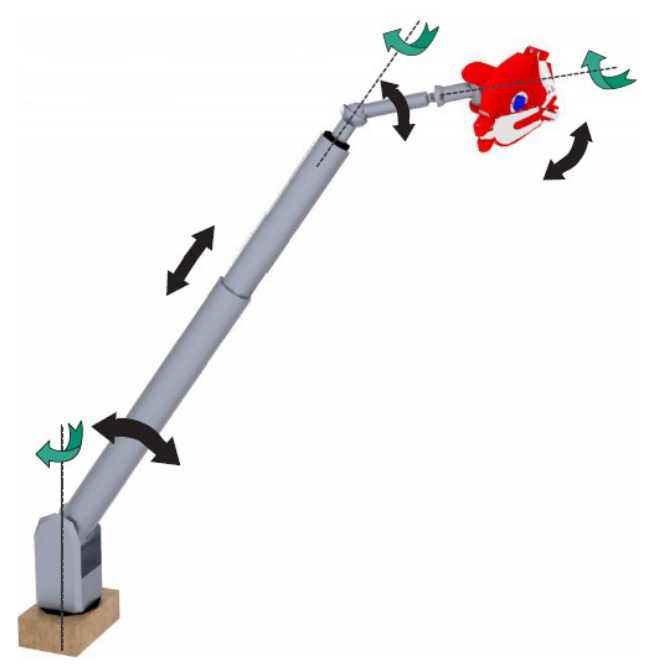

\subsection{Manipulator configuration}

There is a prismatic extension arm which is located at the rear end and it is fixed to the base of the frame. The prismatic extension arm base has a freedom to rotate $360^{\circ}$, but for the application and design purpose the rotation of the base arm is restricted to $180^{\circ}$ and the arm rotates up to $130^{\circ}$ with respect to its axis of rotation. The prismatic extension arm has a primary and secondary arm. The secondary arm moves linearly to the axis of the primary arm. The secondary arm is kept so that the robot need not go so close to the tree 
and to trace coordinates of the fruits which are present in the tree. After the secondary arm, there is a consecutive arm which is a ball and socket joint where the base of the arm rotates $360^{\circ}$ and the arm rotates up to $60^{\circ}$ on all three axes. This consecutive arm is placed in order to by-pass the branches to pick the fruit. Then the end of the arm is connected to the gripper, which rotates about $180^{\circ}$ with respect to its axis of rotation. The rotation of gripper is kept to pluck the fruit from the tree. The detailed layout of the manipulator arm is shown in Figure 2.

\subsection{Gripper design}

The gripper has a three finger like structure which is used to hold the fruit. The base of the gripper is a curved surface and has a camera fixed at its centre to map the fruit for more accuracy in order to hold the fruit. The top of the palm is attached with two finger arms as shown in Figure 3.

Figure 3 Gripper model (see online version for colours)

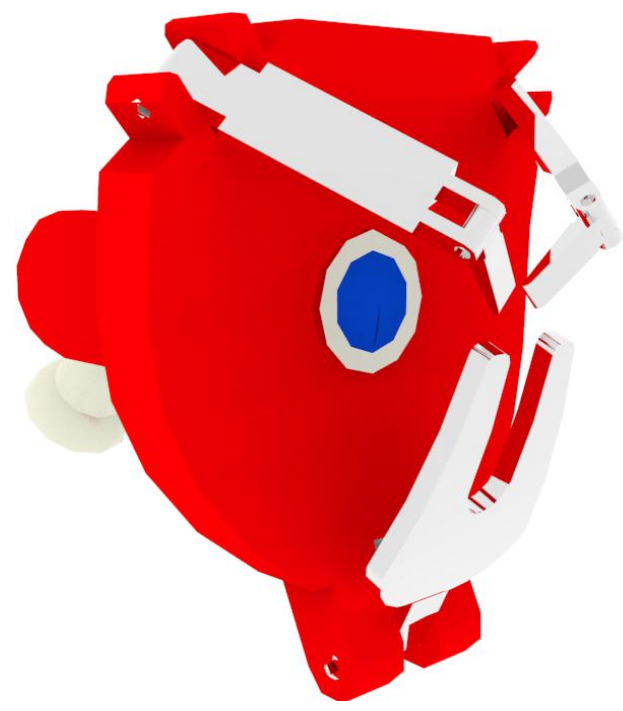

The top two fingers are located $90^{\circ}$ apart from each other. The finger has two parts, the first part rotates in order to arrest the vertical movement of the fruit and the second part rotates in order to provide additional grip such that it restricts the top horizontal movement of the fruit. The flat curved arm from bottom restricts the movement of the lower part of the fruit. These fingers are controlled using feedback obtained from the pressure sensors which are located at the tip of the gripper. In order to pluck the fruit without damaging, it is ensured that the applied pressure exerted on all sides is equal. Once the fruit is plucked, it is dropped into a hopper like structure located below the gripper. The fruit is then directed to the bellows like structure, which falls freely due to gravity and reaches the storage tank at the base. 


\subsection{Bellows design}

A specially designed bellows is made up of a lightweight composition and coated with a flexible material inside; this is to avoid damage that could be caused to the fruit due to rolling down from the hopper. The exterior design of the bellows is shown in Figure 4.

Figure 4 Bellows exterior (see online version for colours)

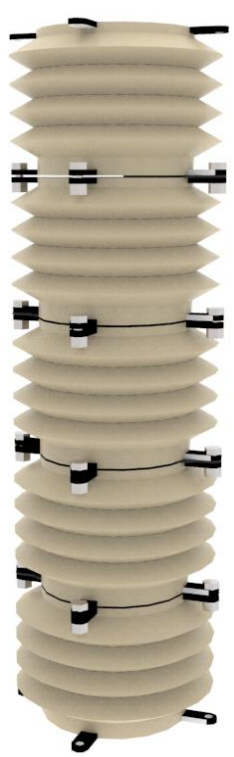

For each section of the bellows a zigzag flexi-pad is placed, this is to further slow down the fruit fall due to gravity and allow it to come down by steps. The flexi-pad is made of elastic-based material so that it could bend easily and return back to its original shape as shown in Figure 5; the bellows also has the property to expand and contract easily. The container and the bellows are connected by a curved tube coated with the same flexible material inside, where the fruit rolls into the container due to gravity.

Figure 5 Bellows interior (see online version for colours)

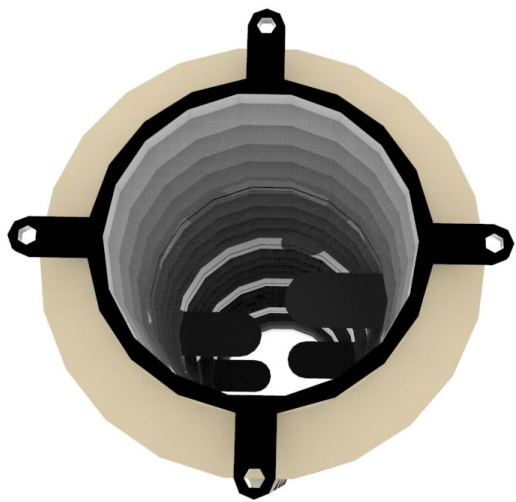




\subsection{Storage container}

A container is mounted on the moving platform of the robot; it is designed in such a way that it can hold a large number of fruits. It is clamped to the base of the vehicle; the fruit rolls and enters the container through the lid. The container has two support rods attached to it on either side. After plucking the fruits and storing it into the container, the vehicle traces its way to the dumper vessel. The dumper vessel has a hydraulic or pneumatic powered arm where it tilts the container with the help of the two support rods. Then the fruits are dumped into the dumper and taken for further process. This dynamically designed robot will increase the production rate and save more time.

\subsection{Sensor and actuator}

Any robotic system must be controlled and actuated as per the desire in order to perform a specific task. This could be done only when we have proper feedback and actuating unit. The feedbacks are collected from the sensors located at various places. There are a group of sensors placed at the gripper, they include vision, collision, pressure and positioning sensors which essentially need to be the most important for the fundamental positioning and locating. The vision sensor locates and maps the fruit accurately and sends this information to the controller, which then actuates the joints to reach the location in an optimal path. The pressure and position sensors serve the purpose of denoting the hold of the fruit. The collision sensor is used to perceive the operating environment in a better way.

\subsection{Vision system}

The main objective of the vision system is to locate the fruit accurately. This is obtained by image processing methods; the location of fruit not only gives a solution to pluck the fruit, but also determines the ability of the manipulator to move to the location with much better accuracy. Owing to the existing natural environmental conditions there may be restrictions in locating and plucking the fruits. Hence these drawbacks are overcome using the colour CCD camera, which captures the original image of the fruit and is processed. There are again the complexity arises due to the background of the image obtained, as it is not possible to get the image of the fruit alone. The presence of branches and leaves further complicates the imaging process. So, any image captured would inevitably have noises mixed with it, by applying a suitable median filter. Median filter not only removes the noise effectively but also preserves the fruit edges and highlights the fruits foreground in a better manner.

The camera is located on the palm of the gripper as this location of the camera makes it more efficient and reliable. There is also another camera located on the base platform which guides the entire vehicle to move in the field without any difficulty. Shortest path algorithms are used which compute the optimal path to reach objective.

\section{Robot kinematics}

In any robotic simulation analysis, the main purpose is to find the relationship between movements of each joint with respect to the others. This could be carried out by doing the 
kinematics analysis. Forward kinematics and the inverse kinematics are the two ways with which we obtain the relationship between the various joints to the end-effectors and vice versa. The forward kinematics is expressed as $F\left(\theta_{1}, \theta_{2}, \theta_{3}, \ldots, \theta_{n}\right)=(x, y, z)$ and the inverse kinematics is represented as $F(x, y, z)=\theta_{1}, \theta_{2}, \theta_{3}, \ldots, \theta_{n}$. Shown in Figure 6 is a simplified block diagram of the robot kinematics. In simple terms knowing the joint relationships and relating it with the end-effectors position and orientation is called forward kinematics, and on the other hand, knowing the position and orientation and relating it with the joint parameters is called inverse kinematics.

Figure 6 Kinematics block diagram

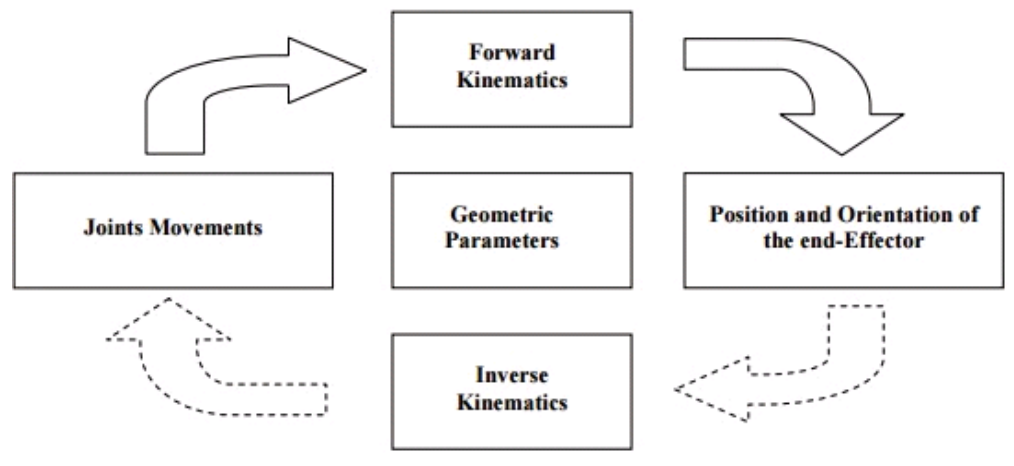

The Denavit-Hartenberg $(\mathrm{DH})$ representation for a robotic manipulator is one of the efficient ways of representing the robot kinematics. The DH representation is the four parameters associated with a particular convention for attaching reference frames to the links of a robot arm. The four parameters of the DH representation are $\alpha_{i-1}$ is the angle between $Z_{i-1}$ and $Z_{i}$ along the $X_{i}$ direction $a_{i-1}$ is the distance between $Z_{i-1}$ and $Z_{i}$ along the $X_{i}$ direction $d_{i}$ is the distance between $X_{i-1}$ and $X_{i}$ along the $Z_{i}$ direction and $\theta_{i}$ is the angle between $X_{i-1}$ and $X_{i}$ along the $Z_{i}$ direction respectively.

The DH table for the fruit harvester is shown in Table 1 . There are totally six joints, out of which the last one is the gripper. Once if we define one coordinate transformation between two frames, where the position and orientation are fixed one with respect to the others it is possible to work with elementary homogeneous transformation operations.

Table 1 DH parameter - robot arm

\begin{tabular}{ccccc}
\hline $\mathrm{i}$ & $\alpha_{i-1}$ & $a_{i-1}$ & $d_{i}$ & $\theta_{i}$ \\
\hline 1 & 0 & 0 & 0 & $\theta_{1}$ \\
2 & 90 & 0 & 0 & $\theta_{2}$ \\
3 & 0 & $\mathrm{a}_{3}$ & 0 & $\theta_{3}$ \\
4 & 0 & $\mathrm{a}_{4}$ & 0 & $\theta_{4}-90$ \\
5 & -90 & 0 & $\mathrm{~d}_{5}$ & $\theta_{5}$ \\
6 & 0 & 0 & 0 & Gripper \\
\hline
\end{tabular}

The values can be obtained from the true measurement of the design, thereby the forward kinematics relationship can be calculated. This yields the following six transformation 
matrices from $T^{1}$ to $T^{6}$ in the following $C_{i}$ and $S_{i}$ are used as conventions for $\cos \theta_{i}$ and $\sin \theta_{i}$.

$$
\begin{aligned}
& T_{1}^{0}=\left(\begin{array}{cccc}
C_{1} & -S_{1} & 0 & 0 \\
S_{1} & C_{1} & 0 & 0 \\
0 & 1 & 0 & d_{1} \\
0 & 0 & 0 & 1
\end{array}\right) \\
& T_{2}^{1}=\left(\begin{array}{cccc}
C_{2} & -S_{2} & 0 & 0 \\
0 & 0 & -1 & 0 \\
S_{2} & C_{2} & 0 & 0 \\
0 & 0 & 0 & 1
\end{array}\right) \\
& T_{3}^{2}=\left(\begin{array}{cccc}
C_{3} & -S_{3} & 0 & a_{3} \\
S_{3} & C_{3} & 0 & 0 \\
0 & 0 & 1 & 0 \\
0 & 0 & 0 & 1
\end{array}\right) \\
& T_{4}^{3}=\left(\begin{array}{cccc}
C_{4} & -S_{4} & 0 & a_{4} \\
S_{4} & C_{4} & 0 & 0 \\
0 & 0 & 1 & 0 \\
0 & 0 & 0 & 1
\end{array}\right) \\
& T_{5}^{4}=\left(\begin{array}{cccc}
C_{5} & -S_{5} & 0 & 0 \\
0 & 0 & 0 & d_{5} \\
S_{5} & C_{5} & 1 & 0 \\
0 & 0 & 0 & 1
\end{array}\right) \\
& T_{\text {Gripper }}=\left(\begin{array}{cccc}
C_{6} & -S_{6} & 0 & 0 \\
S_{6} & C_{6} & 0 & 0 \\
0 & 0 & 1 \\
0 & 0 & 0 & 1
\end{array}\right)
\end{aligned}
$$

The transformations from $T^{1}$ to $T^{6}$ are shown above which are followed to get the overall transformation. The actual joint limits are shown in Table 2.

Table 2 Manipulator arm joint limits

\begin{tabular}{lc}
\hline Joints & Joint limits \\
\hline 1 & $0 \leq \theta_{1} \leq 180^{\circ}$ \\
2 & $60^{\circ} \leq \theta_{2} \leq 60^{\circ}$ \\
3 & $1,200 \leq \mathrm{a}^{3} \leq 1,800$ \\
4 & $0 \leq \theta_{4} \leq 360^{\circ}$ \\
5 & $0 \leq \theta_{5} \leq 360^{\circ}$ \\
Gripper & $-90^{\circ} \leq \theta_{6} \leq 90^{\circ}$ \\
\hline
\end{tabular}

\section{Controller architecture and strategies}

The control architecture of the entire manipulator is shown in Figure 7.

The overview of the control starts with the vision sensor (camera 1) located at the base frame, this camera locates the fruit on its path and transfers the coordinate position 
of those to the processor, the shortest path algorithm generates the optimal path from the base frame to the fruit coordinate. The controller controls all the joint variables so as to move the gripper to the fruit location through a set of transformations. Once the gripper is located in the exact position, the secondary vision sensor (camera 2) located at the gripper facilitates in actuating the control sequence followed in plucking the fruit from the branch by actuating the gripper motors. The gripper also has several other types of sensors which take care of proper plucking of the fruit.

Figure 7 Overall control architecture

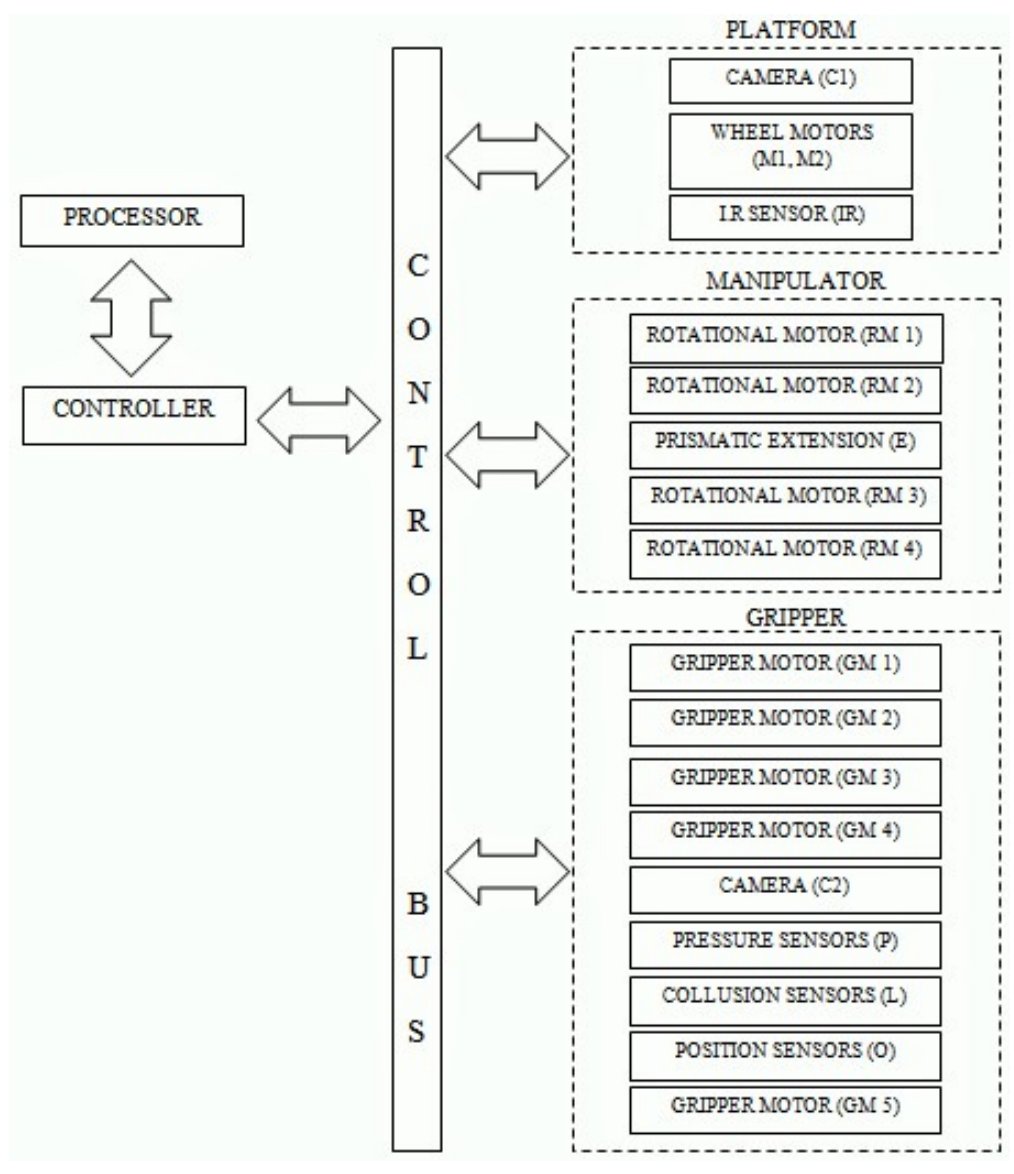

\section{Robot dynamics}

The Newton-Euler expressions are evaluated symbolically for any robotic manipulator, they result the following dynamic equation as in equation (1).

$$
\tau=M(\theta) \theta^{\prime \prime}+V\left(\theta, \theta^{\prime}\right)+G(\theta)
$$

where $M(\theta)$ is the mass matrix of the manipulator with dimension $(n X n), V\left(\theta, \theta^{\prime}\right)$ is the centrifugal and coriolis vectors with dimension $(n X 1)$ and $G(\theta)$ is the gravity vector with 
dimension $(n X 1)$. Each element of $M(\theta)$ and $G(\theta)$ is a complex function that depends on position of all points on the manipulator. The elements of $V\left(\theta, \theta^{\prime}\right)$ are again complex functions that depend on both the position and velocity of all points on the manipulator.

The robot dynamics similar to kinematics have two forms, the forward and inverse dynamics. Known the joint torques and applied forces and estimating the motion of the end-effectors is called the forward dynamics, where as the inverse dynamics we compute the torque from the applied forces and motion.

\section{Trajectory planning}

In general, trajectory planning and tracking are done to find the relationship between the start and end points satisfying the given path constraints mostly in terms of velocity and acceleration. A smooth trajectory is defined using polynomials, thus we have considered a cubic polynomial with four independent coefficient vector as shown below in equation (2). This equation specifies the distance between any points on $t$, eventually from zero to one.

$$
p(t)=a_{3} t^{3}+a_{2} t^{2}+a_{3} t+a_{0}
$$

The corresponding velocity and acceleration are given by equations (3) and (4) respectively.

$$
\begin{aligned}
& p^{\prime}(t)=3 a_{3} t^{2}+2 a_{2} t+a_{1} \\
& p^{\prime \prime}(t)=6 a_{3} t+2 a_{2}
\end{aligned}
$$

On combining the equations and substituting the values of $t$ from zero to one.

Figure 8 Cubic polynomial trajectory (see online version for colours)
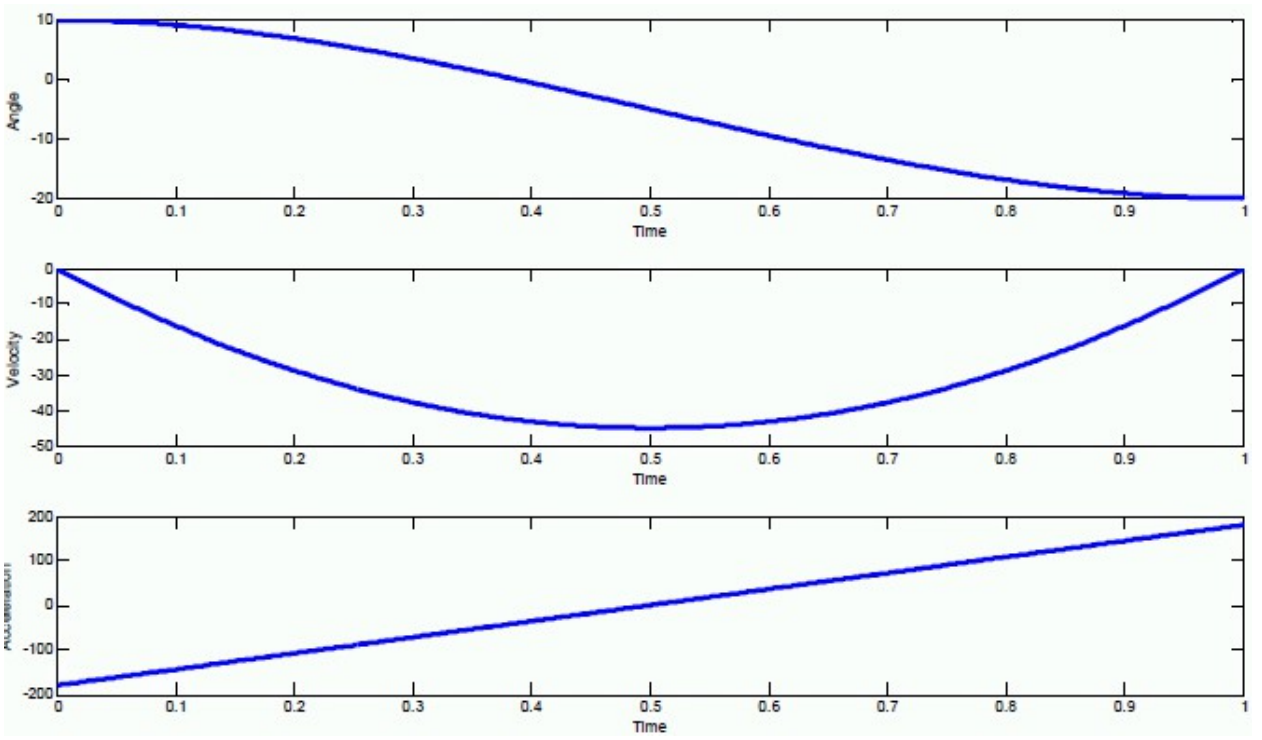
The corresponding degree, velocity and acceleration for a cubic polynomial could be obtained as shown in Figure 8. From the above figure its clear that the position and velocity are continuous at the start point and end point, whereas the acceleration seems to be discontinuous with time, this would lead to a jerk which excites the vibrational modes in the manipulator and results in poor tracking. Hence we must impose constraints on acceleration also, so we consider a quintic polynomial with five independent coefficient vectors as shown below in equation (5).

$$
p(t)=a_{4} t^{4}+a_{3} t^{3}+a_{2} t^{2}+a_{1} t+a_{0}
$$

The corresponding velocity and acceleration are given by equations (6) and (7) respectively.

$$
\begin{aligned}
& p^{\prime}(t)=4 a_{4} t^{3}+3 a_{3} t^{2}+2 a_{2} t+a_{1} \\
& p^{\prime \prime}(t)=12 a_{4} t^{2}+6 a_{3} t+2 a_{2}
\end{aligned}
$$

On combining the equations and substituting the values of $t$ from 0 to 1 , the corresponding degree, velocity and acceleration for a quintic polynomial could be obtained as shown in Figure 9.

Figure 9 Quintic polynomial trajectory (see online version for colours)
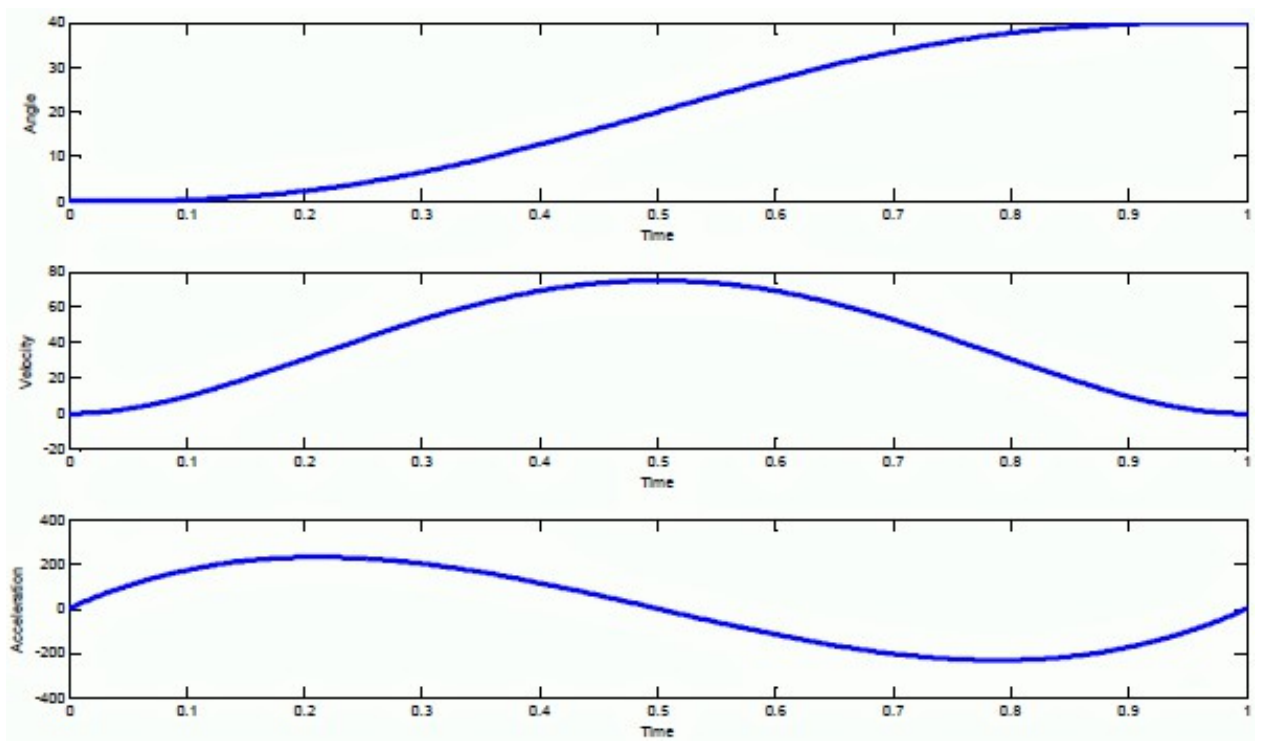

\section{Conclusions}

A conceptual model for a fruit harvesting robot is designed and is explained in detail, the manipulator was conceptualised in all optimistic ways to reduce the functional complexity. The manipulator was designed to harvest fruits in as easy away as possible. The robot kinematics and dynamics were analysed for better understanding of the system 
and to adapt control strategies to the manipulator. This harvesting robot was made to function autonomously using a vision-based control system. Trajectory tracking was carried out to determine the position, velocity and acceleration at various joints. The overall design of the harvesting robot is shown in Figure 10.

The future work of this research is devoted to real time implementation of the system with well adapted control architecture which can compensate for unpredictable external environment. This will increase the speed and accuracy of the system, so that the robot can pluck many fruits in short duration.

Figure 10 Overall model of harvesting robot (see online version for colours)

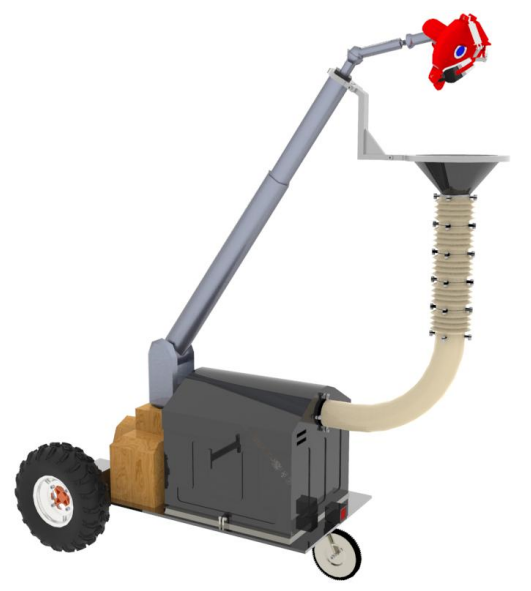

\section{References}

Aljanobi, A.A., Al-hamed, S.A. and Al-Suhaibani, S.A. (2010) 'A setup of mobile robotic unit for fruit harvesting', 19th International Workshop on Robotics in Alpe-Adria Danube Region-RAAD.

De-An, Z., Jidong, L., Wei, J., Ying, Z. and Yu, C. (2011) 'Design and control of an apple harvesting robot', $S D$ Biosystems Engineering, Vol. 110, pp.112-122.

Edan, Y. and Miles, G.E. (1994) 'Systems engineering of agricultural robot design', IEEE Transactions on Systems, and Cybernetics, August, Vol. 24, pp.1259-1265.

Edan, Y., Flash, T., Peiper, U., Shmulevich, I. and Sarig, Y. (1991) 'Near-minimu-time task planning for fruit-picking robots', IEEE Transactions on Robotics and Automation, February, Vol. 7, pp.48-56.

Hamrita, T.K., Tollner, E.W. and Schafer, R.L. (2000) 'Toward fulfilling the robotic farming vision: advances in sensors and controllers for agricultural application', IEEE Transactions on Industry Applications, Vol. 36, pp.1026-1032.

Kondo, N. (2003) 'Fruit grading robot', Proceeding of IEEE Applied Intelligent Mechatronics, pp.1366-1371.

Li, L.X. and Shi, C.T. (2010) 'Path planning of fruit picking robot based on genetic algorithm', CMCE 2010 International conference on Computer, Mechatronics, pp.248-251.

Li, P., Lee, S-H. and Hsu, H-Y. (2011a) 'Study on Citrus Fruit image data separability by segmention method', Procedia Engineering, Vol. 23, pp.408-416.

Li, P., Lee, S-H. and Hsu, H-Y. (2011b) 'Review on fruit harvesting method for potential use of automatic fruit harvesting systems', Procedia Engineering, Vol. 23, pp.351-366. 
Optimal conceptual design and vision-based control of a fruit harvesting robot 59

Sanders, K.F. (2005) 'Orange harvesting system review', Biosystems Engineering and Agricultural Research Centre, Vol. 90, pp.115-124.

Suprem, A., Mahalik, N. and Kim, K. (2013) 'Computer standards and interfaces', Computer Standards and Interfaces, Vol. 35, pp.355-364. 